\title{
OPEN A magnetic sensor using a 2D van der Waals ferromagnetic material
}

\author{
Valery Ortiz Jimenez ${ }^{1}$, Vijaysankar Kalappattil ${ }^{1}$, Tatiana Eggers ${ }^{1}$, Manuel Bonilla ${ }^{1}$, \\ Sadhu Kolekar ${ }^{1}$, Pham Thanh Huy ${ }^{2}$, Matthias Batzill ${ }^{1}$ \& Manh-Huong Phan ${ }^{1 *}$
}

Two-dimensional (2D) van der Waals ferromagnetic materials are emerging as promising candidates for applications in ultra-compact spintronic nanodevices, nanosensors, and information storage. Our recent discovery of the strong room temperature ferromagnetism in single layers of $\mathrm{VSe}_{2}$ grown on graphite or $\mathrm{MoS}_{2}$ substrate has opened new opportunities to explore these ultrathin magnets for such applications. In this paper, we present a new type of magnetic sensor that utilizes the single layer $\mathrm{VSe}_{2}$ film as a highly sensitive magnetic core. The sensor relies in changes in resonance frequency of the LC circuit composed of a soft ferromagnetic microwire coil that contains the ferromagnetic $\mathrm{VSe}_{2}$ film subject to applied DC magnetic fields. We define sensitivity as the slope of the characteristic curve of our sensor, $d f_{0} / d H$, where $f_{0}$ is the resonance frequency and $H$ is the external magnetic field. The sensitivity of the sensor reaches a large value of $16 \times 10^{6} \mathrm{~Hz} / \mathrm{Oe}$, making it a potential candidate for a wide range of magnetic sensing applications.

Since intrinsic long-range ferromagnetic order was realized in 2017 with bulk exfoliated monolayers of $\mathrm{Cr}_{2} \mathrm{Ge}_{2} \mathrm{Te}_{6}$ and $\mathrm{CrI}_{3}{ }^{1,2}$, the potential of two-dimensional (2D) van der Waals magnets has excited the scientific community $^{3-11}$. While these $2 \mathrm{D}$ magnets have demonstrated their usefulness in magnetoelectric devices, their technological applications are restricted to low temperatures $(<100 \mathrm{~K})^{6-10}$. In contrast, recent discoveries of the room temperature (RT) ferromagnetism in transition metal dichalcogenide (TMD) monolayers of $\mathrm{VSe}_{2}$ and $\mathrm{MnSe}_{2}$ grown by van der Waals epitaxy on various substrates (graphite, $\mathrm{MoS}_{2}$, GaSe) may enable applications at ambient temperature ${ }^{3,4,10}$. Due to the nature of atomically thin materials, their physical properties are sensitive to external stimuli. In this regard, it would be very interesting to exploit the potential of $2 \mathrm{D}$ magnets for magnetic sensing applications. In this paper, we present a new type of magnetic sensor that integrates a single layer $\mathrm{VSe}_{2}$ film within a Co-rich microwire-based coil.

Induction coil sensors have been widely used due to the simplicity of their construction and a well known transfer function ${ }^{12}$. It is established that the sensivity of an induction coil is limited by the number of windings; the greater the number of turns the higher the sensitivity. This quickly becomes a problem for modern applications where it is desirable to limit the size of sensors. Adding a soft ferromangetic core with high relative permeability can largely increase the sensitivity of a coil, and hence allow for smaller sensor sizes ${ }^{13}$. A similar working principle has recently been used in the design of magnetic microwire coil-LC resonator sensors, but cobalt rich magnetic microwires are used instead of non-magnetic conductors such as copper ${ }^{14}$. The working principle of the sensor reported in this paper is fundamentally different than that of a conventional induction coil sensor. It relies on the changes in resonance frequency caused by external magnetic fields, instead of simply measuring the induction of the coil. It is also different from the magnetic microwire coil-LC resonator sensor ${ }^{14}$ that relies in changes in impedance of the microwire caused by external magnetic fields.

A simple model for a coil sensor is a lumped element representation of a non-ideal inductor. Winding cylindrical conductors close to each other will introduce parasitic elements $R_{p a r}$ and $C_{p a r}$, such that the non-ideal inductor can be represented as a series combination of an ideal inductor $L$ and $R_{p a r}$, in parallel with $C_{p a r}{ }^{15-17}$. The impedance of the coil $Z_{\text {Coil }}$ can then be written as

$$
Z_{\text {coil }}=\frac{R_{p a r}+j \omega\left[\mathrm{L}\left(1-\omega^{2} \mathrm{LC}_{p a r}\right)-C_{\mathrm{par}} R_{p a r}^{2}\right]}{\left(1-\omega^{2} \mathrm{~L} C_{p a r}\right)^{2}+\left(\omega C_{p a r} R_{p a r}\right)^{2}}
$$


a

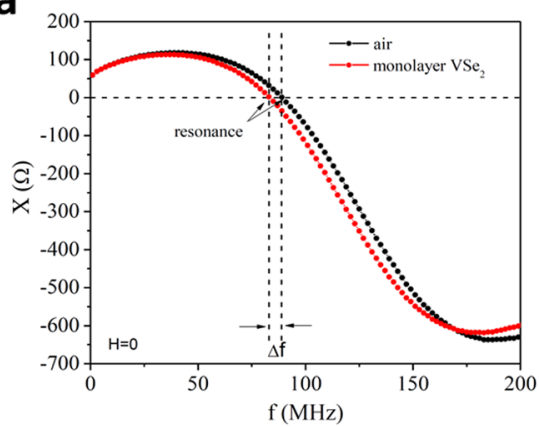

b
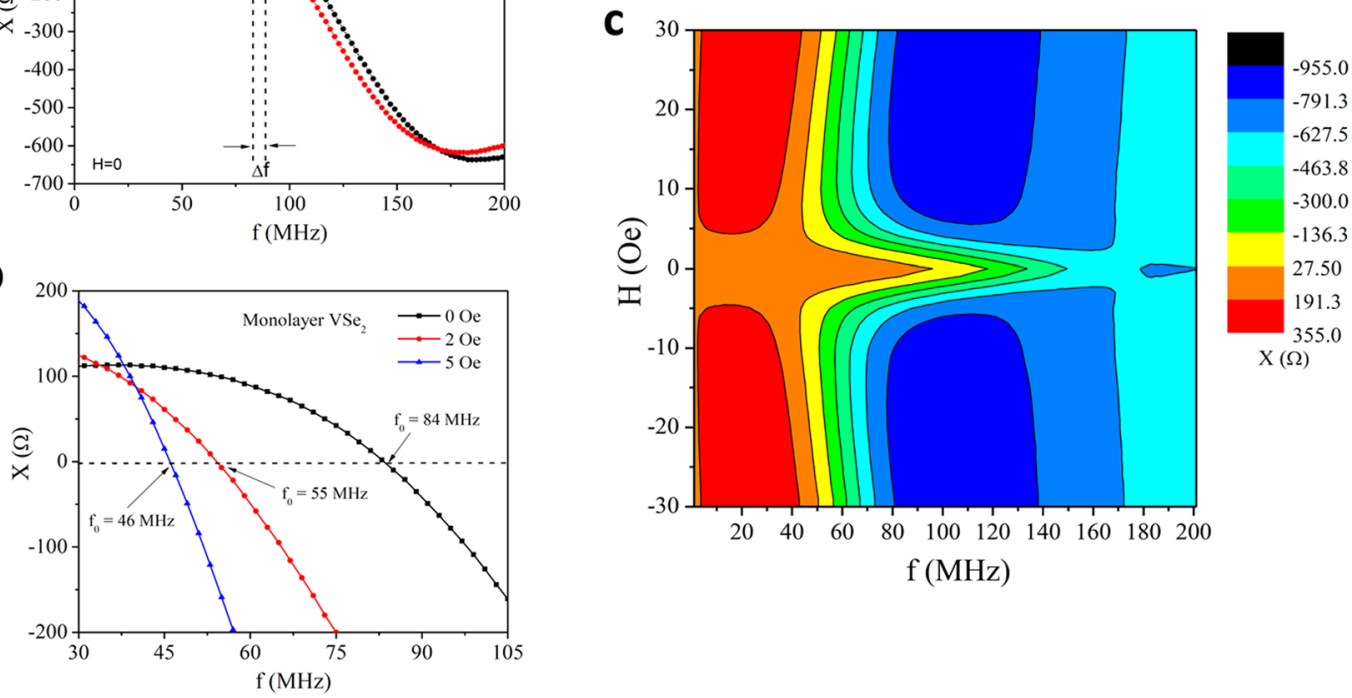

Figure 1. (a) Frequency dependence of the reactance of the sensor with and without the magnetic $\mathrm{VSe}_{2}$ film in the absence of an external magnetic field; (b) Frequency dependence of the reactance of the sensor with the magnetic $\mathrm{VSe}_{2}$ film shows large shifts in the resonant frequency with respect to applied magnetic fields; (c) 2D surface plot shows the magnetic field and frequency dependences of reactance.

where $\omega$ is the angular frequency, and $j$ is the imaginary unit. Resonance will occur when the inductive reactance $\left(X_{L}\right)$ and the capacitive reactance $\left(X_{C}\right)$ are equal in magnitude but differ in phase by 180 degrees. At this point very little current flows through the wire, the impedance $Z_{\text {Coil }}$ becomes very large and self-resonance is achieved ${ }^{18,19}$. The resonance frequency, $f_{0}$, is given by

$$
f_{0}=\frac{\sqrt{1-\left(\mathrm{R}_{\mathrm{par}}^{2} \mathrm{C}_{\mathrm{par}} / \mathrm{L}\right)}}{2 \pi \sqrt{\mathrm{LC}_{\mathrm{par}}}} .
$$

We expect a self-resonant behavior in the microwire coil as well, but the resonance frequency will be different than that of the inductor since the wire is now a magnetic material. We must also consider the effects of a ferromagnetic core on the sensor. The core will modify the relative permeability in the space within the coil, and this will in turn change the flux through the coil and hence affect the inductance. Since the permeability is field dependent, an external magnetic field will modify the permeability of the core and the resonance frequency with it. Additionally, we must consider that the wire itself is magnetic, which will lead to an effective permeability of the microwire and the core. The inductance of the sensor must then depend on this effective permeability, $L=L\left(\mu_{\text {eff }}\right)$.The sensitivity of the sensor is defined as the rate of change of the resonance frequency with respect to the external DC magnetic field,

$$
\text { Sensitivity }=\frac{d f_{0}}{d H}
$$

The $Q$ factor is also calculated by measuring the bandwidth, BW, and the resonance frequency using the following relation,

$$
Q=\frac{f_{0}}{B W}
$$

\section{Results and Discussion}

A set of measurements is performed to characterize the sensor; the resonance frequency is found for every value of the field. Resonance is determined by the zero crossing of the reactance. To see the effects of the monolayer core we show in Fig. 1(a) the reactance of the coil with and without the monolayer core at zero field. The presence of the monolayer shifts the resonance frequency of the sensor by a few megahertz. We then applied the external field and observed how the reactance curve is shifted along with the corresponding resonance frequency as shown in 
a

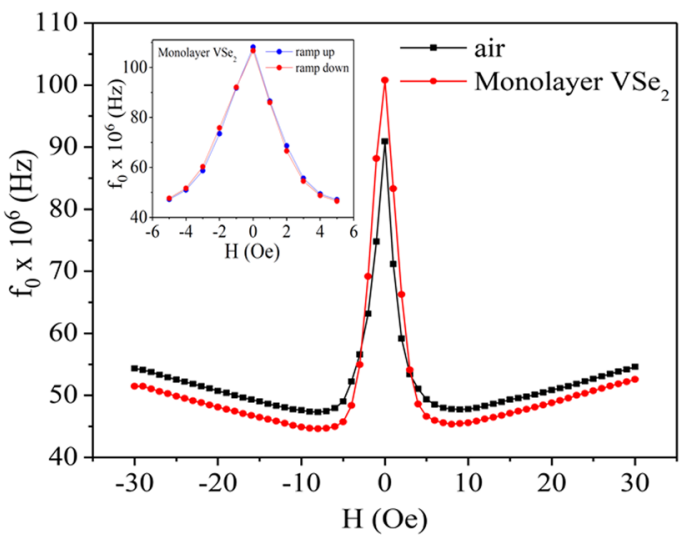

b

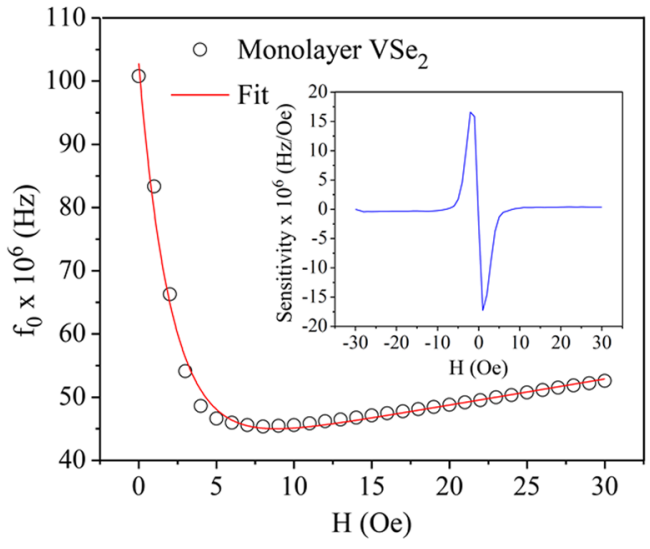

Figure 2. (a) The resonant frequency of the sensor $\left(f_{0}\right)$ changes as a function of magnetic field. A large change is achieved at fields between 0 and $5 \mathrm{Oe}$. A comparison between the air coil and the coil with the monolayer $\mathrm{VSe}_{2}$ core is shown. The inset shows that hysteresis in the sensor is negligible in the low field regime (below $5 \mathrm{Oe}$ ); (b) a fit of the magnetic field dependent resonant frequency $f_{0}(H)$ data is displayed, with the inset showing the magnetic field dependence of the sensor sensitivity.

Fig. 1(b), for the coil with the monolayer in its core. The reactance of the coil being proportional to the inductance changes drastically with frequency and applied field, as well as the resonance frequency (see Fig. (1c)).

The resonance frequency is determined for different values of an externally applied magnetic field, between -30 to 30 Oe. Figure 2(a) shows the change in resonance frequency as a function of applied field. Unique values of the resonance frequency exist for fields smaller than $5 \mathrm{Oe}$. A challenge we must consider when using a magnetic material is the hysteresis that is inherent to it. We have found that hysteresis in the sensor is negligible as long as fields no greater than $5 \mathrm{Oe}$ are applied (see the inset of Fig. 2(a)). Since the sensor is intended to detect small magnetic fields, this should not hinder the sensor's performance. At higher fields, the magnetic wire saturates, and $f_{0}$ changes very slowly, rending the sensor inoperable. A fit of the right branch of the $f_{0}$ plot is shown in Fig. 2(b) with the following equation,

$$
f_{0}=a+b H+c * r^{H},
$$

where $f_{0}$ is the resonance frequency, $H$ is the external magnetic field and $a=40.50, b=0.41, c=62.30$ and $r=0.62$ are fitting parameters. When compared to the behavior of the air coil, the parameter $c$ increases when the core is inserted; it is the parameter that depends the most on the properties of the core, hence modifying the contribution from the exponential term to the change in resonance frequency. While the wire dominates the behavior of the sensor, a magnetic core may be used to improve sensitivity. The sensitivity of the sensor is shown in the inset of Fig. 2(b). Values as large as $16 \times 10^{6} \mathrm{~Hz} / \mathrm{Oe}$ are achieved for the sensitivity. Future work must be focused on determining the minimum detectable signal of the sensor, but the high sensitivity may allow for applications such as bio-detection, where magnetic nanoparticles are used as bio-markers for pathogen detection and quantifying low particle concentrations is neccessary ${ }^{20}$. We also show how the $\mathrm{VSe}_{2}$ monolayer compares with a commercially available METGLAS 2714A ribbon (see Fig. S1). These ribbons have ultra-high DC permeability and are known to be excellent magnetic cores. The METGLAS ribbon shows a larger improvement in sensitivity than the single layer of $\mathrm{VSe}_{2}$. This is expected from a soft bulk material, but it shows that even a single layer of $\mathrm{VSe}_{2}$ can serve as a magnetic core to improve sensitivity of coil-based sensors, particularly in the low field regime.

Finally, we have determined the Q factor of the sensor at different values of external DC magnetic field, as shown in Fig. 3. We have found that for very small fields, smaller than 1 Oe, the Q factor is 0.6 ; as the field increases it reaches a minimum value of 0.36 at 3 Oe. Most of the losses are associated with the magnetic wire itself; the core slightly decreases the Q factor by less than 0.1 (see Fig. S2). This is consistent with the soft ferromagnetic characteristic of monolayer $\mathrm{VSe}_{2}$ (Fig. 4(b)), which predicts minimal magnetic losses.

In summary, a magnetic field sensor was built using a magnetic microwire coil as the sensing element with a single layer $\mathrm{VSe}_{2}$ film as the core. Sensitivity as large as $16 \times 10^{6} \mathrm{~Hz} / \mathrm{Oe}$ for very small fields was obtained. While conventional bulk materials with ultra-high permeability show a higher increment in the sensitivity of coil-based sensors, a single layered 2D magnet can still increase the sensor performance to a comparable level. Furthermore, core losses were shown to be minimal which is desirable for sensor design.

\section{Methods}

To make the magnetic sensor, $\mathrm{a} \mathrm{Co}_{69.25} \mathrm{Fe}_{4.25} \mathrm{Si}_{13} \mathrm{~B}_{12.5} \mathrm{Nb}_{1}$ wire (diameter, $\sim 60 \mu \mathrm{m}$ ) was wound into a 15-turn, $10 \mathrm{~mm}$ long coil with a $5 \mathrm{~mm}$ internal diameter. The fabrication details and material characterization of the microwire can be found elsewhere ${ }^{21,22}$. Monolayer $\mathrm{VSe}_{2}$ was used as the core of the coil. The single layer films of $\mathrm{VSe}_{2}$ were grown on graphite (HOPG) and single crystal $\mathrm{MoS}_{2}$ by molecular beam epitaxy (MBE); the details of which have been reported in our previous work ${ }^{3}$. Since both monolayer $\mathrm{VSe}_{2}$ samples grown on $\mathrm{MoS}_{2}$ and HOPG show similar magnetic and sensing properties at ambient temperature, in this paper we only report on the properties of 


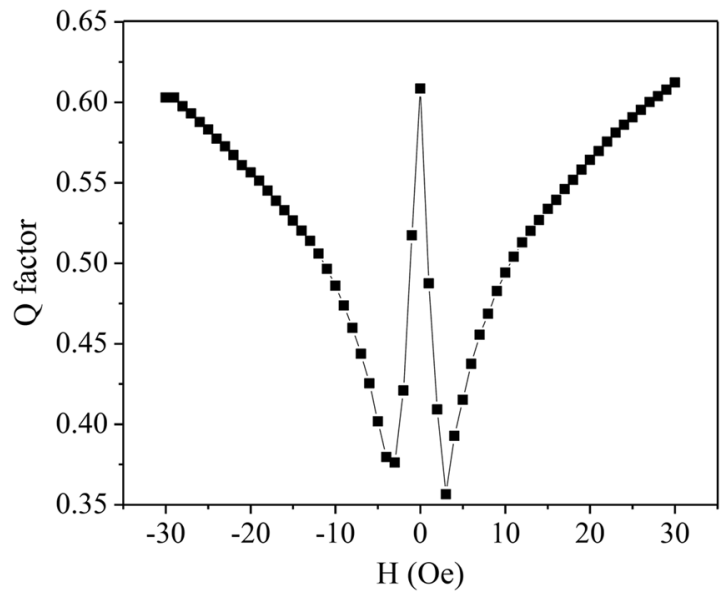

Figure 3. Magnetic field dependence of the $Q$ factor of the sensor. The significant change in $Q$ is observed at low field regime.

a
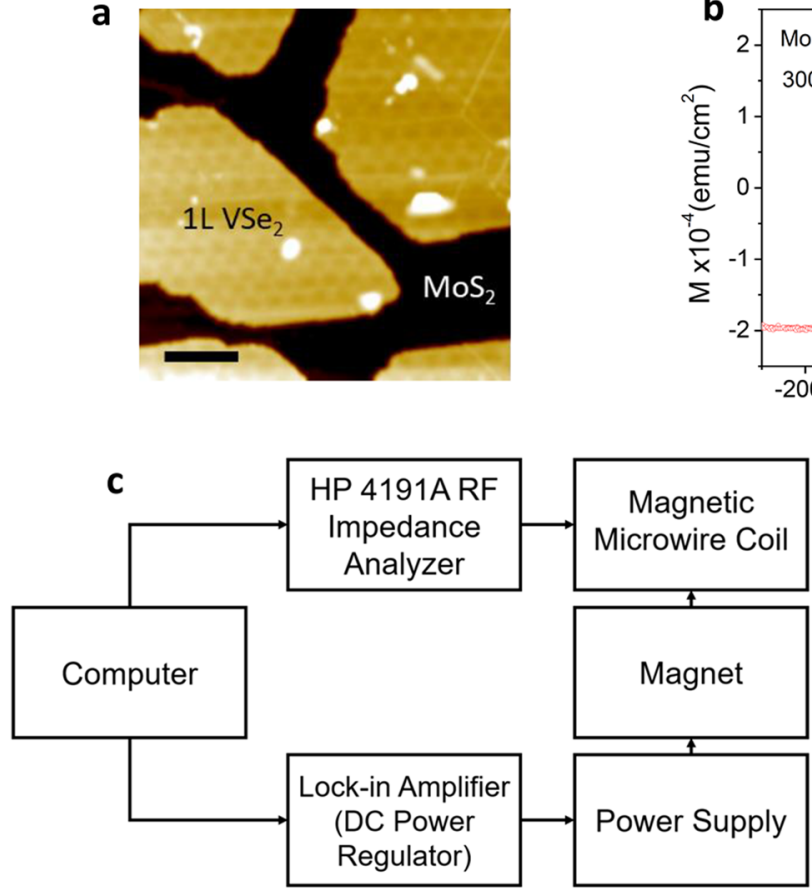

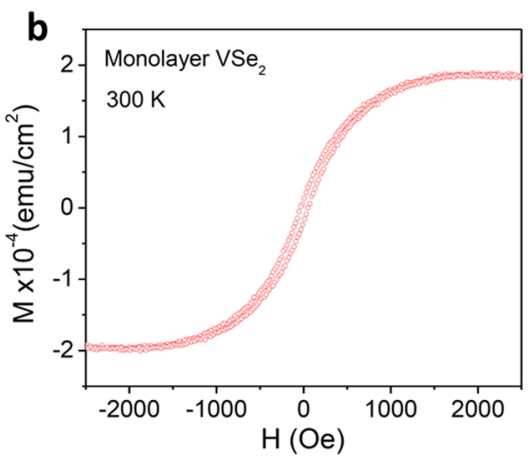

d

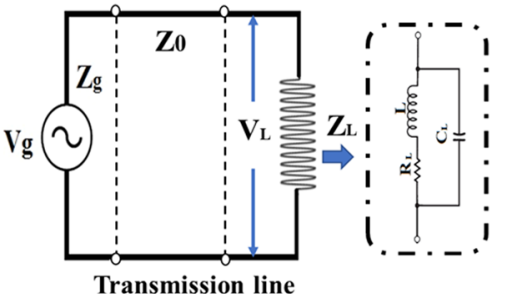

Figure 4. (a) A typical STM image of the single layer $\mathrm{VSe}_{2}$ film grown on single crystal $\mathrm{MoS}_{2}$; (b) the in-plane magnetic hysteresis $(M-H)$ loop of the film measured at $300 \mathrm{~K}$; (c) A block diagram of the measurement setup for testing performances of the sensor; (d) the LC-circuit composed of a magnetic microwire with the $\mathrm{VSe}_{2}$ film inserted in it.

monolayer $\mathrm{VSe}_{2}$ grown on $\mathrm{MoS}_{2}$, but the sensor characterization data for monolayer $\mathrm{VSe}_{2}$ grown on HOPG can be found in Fig. S1. We note that HOPG is diamagnetic in nature and has no appreciate change in response to magnetic field ${ }^{3}$. Figure 4(a) shows a typical scanning tunnel microscopy (STM) image of the $\mathrm{VSe}_{2}$ film. It can be seen that the single layer of $\mathrm{VSe}_{2}$ was epitaxially grown on the $\mathrm{MoS}_{2}$ substrate. The magnetization versus magnetic field $(M-H)$ curves, measured by a vibrating sample magnetometer (VSM) on VSe 2 grown on both HOPG and $\mathrm{MoS}_{2}$, show a soft ferromagnetic characteristic (small coercive field, small remanent magnetization, and high saturation magnetization) of the single layer $\mathrm{VSe}_{2}$ film at room temperature (Fig. 4(b)), which is desirable for its use as the core of the sensor that itself operates at room temperature. Sensor characterization and magnetic measurements were performed by applying an in-plane magnetic field, along the easy axis of the monolayer $\mathrm{VSe}_{2}$. The core was $\sim 14 \mathrm{~mm}$ long and $\sim 4 \mathrm{~mm}$ wide, allowing us to insert and remove the core with ease. The sensor was mounted on a test fixture made of a dielectric material on top of a copper ground plane; SMA connector ports were soldered to the ground plane on two ends and the two leads of the inductor were soldered to the center pin of each connector. 
One port reflection measurements were performed with an HP 4191A impedance analyzer over the frequency range 1-200 MHz. A simple short-open-load (SOL) calibration was made. The test fixture was connected to the impedance analyzer through a coaxial cable, and it was terminated with a $50 \mathrm{ohm}$ cap. The effects of the cable were removed by the calibration. The impedance $(Z)$, resistance $(R)$, and reactance $(X)$ are calculated from the reflection measurement. An external DC magnetic field was generated by a Helmholtz coil. A diagram of the setup is shown in Fig. 4(c,d). The external field was applied transverse to the coil axis and in plane of the 2D magnet. A frequency sweep was performed for every value of the field which was swept from -30 to 30 Oe in steps of 1 Oe.

\section{Data availability}

The datasets generated during and/or analyzed during the current study are available from the corresponding author on reasonable request.

Received: 21 June 2019; Accepted: 17 February 2020;

Published online: 16 March 2020

\section{References}

1. Huang, B. et al. Layer-dependent ferromagnetism in van der Waals crystal down to the monolayer limit. Nature 546, 270 (2017)

2. Gong, C. et al. Discovery of intrinsic ferromagnetism in two-dimensional can der Waals crystals. Nature 546, 265 (2017).

3. Bonilla, M. et al. Strong room-temperature ferromagnetism in VSe ${ }_{2}$ monolayers on van der Waals substrates. Nature Nanotechnol. 13, 289 (2018).

4. O'Hara, D. J. et al. Room Temperature Intrinsic Ferromagnetism in Epitaxial Manganese Selenide Films in the Monolayer Limit. Nano Lett. 18, 3125 (2018).

5. Deng, Y. et al. Gate-tunable room-temperature ferromagnetism in two-dimensional $\mathrm{Fe}_{3} \mathrm{GeTe}_{2}$. Nature 563, 94-99 (2018).

6. Jiang, S., Shan, J. \& Mak, K. F. Electric-field switching of two-dimensional van der Waals magnets. Nat. Mater. 17, 406-410 (2018).

7. Huang, B. et al. Electrical control of 2D magnetism in bilayer $\mathrm{CrI}_{3}$. Nat. Nanotechnol. 13, 544 (2018).

8. Song, T. C. et al. Gian tunneling magnetoresistance in spin-filter van der Waals heterostructures. Science, https://doi.org/10.1126/ science.aar4851 (2018).

9. Umar Farooq, M. \& Hong, J. Switchable valley splitting by external electric field effect in graphene/CrI ${ }_{3}$ heterostructures. npj $2 \mathrm{D}$ Materials and Applications 3:3 (2019).

10. 2D magnetism gets hot, Nature Nanotechnology 13, 269 (2018).

11. Burch, K. S., Mandrus, D. \& Park, J. G. Magnetism in two-dimensional van der Waals materials. Nature 563, 47 (2018).

12. Tumanski, S. Induction coil sensors-a review. Meas. Sci. Technol. 18 R31 (2007).

13. Devkota, J. et al. A soft ferromagnetic multiwire-based inductance coil sensor for sensing applications. J. Appl. Phys. 116, 234504 (2014).

14. Thiabgoh, O., Eggers, T. \& Phan, M. H. Sensors and Actuators A 265, 120 (2017).

15. Grandi, G., Kazimierczuk, M. K., Massarini, A. \& Reggiani, U. Stray capacitances of single-layer solenoid air-core inductors. IEEE Trans. Ind. Appl. 35, 1162 (1999).

16. Paul, C. R. Introduction to electromagnetic compatibility, second ed., John-Wiley, New Jersey, (2006).

17. Floyd, T. L. \& Buchla, D. Electronics Fundamentals: Circuits, Devices \& Applications, eight ed., Prentice Hall Press, New Jersey, (2009).

18. Wangsness, R. K. Electromagnetic fields, second ed., John-Wiley \& Sons, New York, (1986).

19. Scherz, P. Practical electronics for inventors, second ed., McGraw-Hill, New York, (2000).

20. Giouroudi, I. \& Kokkinis, G. Recent Advances in Magnetic Microfluidic Biosensors. Nanomaterials 7, 171 (2017).

21. Shen, H. et al. Optimization of mechanical and giant magneto-impedance (GMI) properties of melt-extracted Co-rich amorphous microwires. Phys. Status. Solidi. A 211, 1668 (2014).

22. Wang, H., Xing, D., Wang, X. \& Sun, J. High Performance Soft Magnetic Materials. Metall. Mater. Trans. A 42A, 1103-1108 (2010).

\section{Acknowledgements}

Work was supported by the VISCOSTONE USA under Grant No. 1253113200, the US Department of Energy, Office of Basic Energy Sciences, Division of Materials Sciences and Engineering under Grant No. DE-FG0207ER46438 (magnetic studies), and the National Science Foundation under grants DMR-1701390 and ECCS1608654. T.E. also acknowledges support from NASA Florida Space Grant Consortium (FSGC) under Award No. 1253-1124-00.

\section{Author contributions}

V.O.J., T.E., P.T.H. and M.H.P. initiated the concept. V.O.J. designed, fabricated and tested the sensor. V.K. performed magnetic measurements. The $\mathrm{VSe}_{2} / \mathrm{MoS}_{2}$ and $\mathrm{VSe}_{2} / \mathrm{HOPG}$ films were grown by $\mathrm{MBE}$ and structurally characterized by M.B., S.K. and M.B. V.O.J. wrote the manuscript with inputs from all other authors. M.H.P. led the research project.

\section{Competing interests}

The authors declare no competing interests.

\section{Additional information}

Supplementary information is available for this paper at https://doi.org/10.1038/s41598-020-61798-2.

Correspondence and requests for materials should be addressed to M.-H.P.

Reprints and permissions information is available at www.nature.com/reprints.

Publisher's note Springer Nature remains neutral with regard to jurisdictional claims in published maps and institutional affiliations. 
(c) (i) Open Access This article is licensed under a Creative Commons Attribution 4.0 International License, which permits use, sharing, adaptation, distribution and reproduction in any medium or format, as long as you give appropriate credit to the original author(s) and the source, provide a link to the Creative Commons license, and indicate if changes were made. The images or other third party material in this article are included in the article's Creative Commons license, unless indicated otherwise in a credit line to the material. If material is not included in the article's Creative Commons license and your intended use is not permitted by statutory regulation or exceeds the permitted use, you will need to obtain permission directly from the copyright holder. To view a copy of this license, visit http://creativecommons.org/licenses/by/4.0/.

(C) The Author(s) 2020 